\title{
New formalism for self-consistent parameters optimization of highly efficient solar cells
}

\author{
A.V. Sachenko ${ }^{1}$, V.P. Kostylyov ${ }^{1}$, M.R. Kulish ${ }^{1}$, I.O. Sokolovskyi ${ }^{1}$, A.I. Shkrebtii ${ }^{2}$ \\ ${ }^{I} V$. Lashkaryov Institute of Semiconductor Physics, NAS of Ukraine \\ 41, prospect Nauky, 03028 Kyiv, Ukraine \\ ${ }^{2}$ University of Ontario Institute of Technology, Oshawa, ON, Canada \\ E-mail:sach@isp.kiev.ua
}

\begin{abstract}
We analyzed self-consistently photoconversion efficiency of direct-gap $\mathrm{A}^{3} \mathrm{~B}^{5}$ semiconductors based solar cells and optimized their main physical characteristics. Using gallium arsenide (GaAs) as an example and new efficient optimization formalism, we demonstrated that commonly accepted light re-emission and reabsorption in solar cells in technologically produced GaAs (in particular, with solid- or liquid-phase epitaxy) are not the main factors responsible for high photoconversion efficiency. As we proved instead, the doping level of the base material and its doping type as well as Shockley-Read-Hall and surface recombination velocities are much more important factors responsible for this photoconversion. We found that the maximum photoconversion efficiency (about $27 \%$ for AM1.5 conditions) in GaAs with typical parameters of recombination centers can be reached for $p$-type base doped at $2 \cdot 10^{17} \mathrm{~cm}^{-3}$. The open-circuit voltage $V_{O C}$ formation features are analyzed. The optimization provides a significant increase in $V_{O C}$ and the limiting photoconversion efficiency close to $30 \%$. The approach of this research allows to predict the expected solar cell (for both direct- and indirect-gap semiconductor) characteristics, if material parameters are known. The applied formalism allows to analyze and to optimize mass production of both tandem solar cell and one-junction SC parameters.
\end{abstract}

Keywords: solar cell, efficiency, radiative recombination, saturation currents, doping, capture cross-section.

Manuscript received 16.01.14; revised version received 02.04.14; accepted for publication 12.06.14; published online 30.06.14.

\section{Introduction}

In recent years, an approach to the modeling of the tandem solar cells (TSC) limit photoconversion efficiency that considers photon recycling (re-emission and reabsorption of photons) in structures with multiple absorption of light as the key point was developed (see, e.g., $[1,2])$. A point of view that there is a strong correspondence between the internal quantum yield of luminescence $q_{p l}$ and photoconversion efficiency $\eta$ (for defined model of the light absorption in the structure) is presented in these researches. The photoconversion efficiency in GaAs was calculated in papers $[1,2]$ as a confirmation of this concept. This material is not chosen by chance. On the one hand, GaAs is the key semiconductor when creating TSC based on direct-gap semiconductors of $\mathrm{A}^{3} \mathrm{~B}^{5}$ group. On the other hand, in double heterostructures AlGaAs-GaAs-AlGaAs the internal luminescence quantum yield is close to $100 \%$ under certain conditions, as it was shown experimentally [3]. Therefore, the influence of reabsorption and re-emission of light on the efficiency 
of solar cells based on GaAs was announced as very significant $[1,2]$.

However, as will be shown below, idealized assumptions used in $[1,2]$ are not being implemented in practice, and the implementation of typical semiconductor solar cell parameters are unsuitable for calculating and optimizing the parameters of a solar cell. Let us consider these assumptions in more detail. The basic assumption is the neglect of nonradiative recombination channels. The internal quantum yield of luminescence close to $100 \%$ is assumed. In fact, this value of $q_{p l}$ was obtained in the laser structure with a high excitation level $\left(\sim 10^{17} \mathrm{~cm}^{-3}\right)$ [3]. In standard operation SC conditions, excitation is several orders of magnitude smaller. It leads to a substantial decrease in the internal quantum efficiency of luminescence. To prove it, we obtained, solved and analyzed in details the equation for the $q_{p l}$ magnitude that is valid for an arbitrary excitation level and takes into account basic mechanisms of recombination.

Next approximation $[1,2]$ is the assumption that the light absorption coefficient $\alpha$ in high-purity gallium arsenide (doping level of semiconductor $\leq 10^{14} \mathrm{~cm}^{-3}$ ) can be used for calculation of the short-circuit current and open-circuit voltage. In fact, as shown in this paper, even if implemented currently longest in GaAs Shockley-Read-Hall lifetimes $\left(\sim 10^{-6} \mathrm{~s}\right)$, to get high values of the open-circuit voltage, it is necessary to implement the doping level of semiconductor $\geq 10^{17} \mathrm{~cm}^{-3}$. Thus, as shown in [5], the light absorption coefficient of doped GaAs is substantially different from that of the high-clean GaAs. As a result, the short-circuit current in $p$-type semiconductor increases and in $n$-type semiconductor decreases. Calculation of short-circuit current expressions for the internal quantum efficiency $q_{S C}$ for cases where the diffusion length $L$ significantly exceeds the SC thickness $d$, while the surface recombination velocity $S$ is zero, was made $[1,2]$. In this paper, much more general expression for $q_{S C}$ is used for plane-parallel structures. It is valid in particular for arbitrary ratio between $L$ and $d$, for arbitrary $S$ and for any value of the light reflectance on the back surface $R_{d}$ varying from 0 to 1 .

Ding et al. [4] applied a more general approach $[1,2]$. Calculation of the GaAs photoconversion efficiency [4] takes into account recombination in the space charge region (SCR) in recombination current calculation as well as the reabsorption and re-emission of photons. The saturation current $I_{0 r}$ is introduced for the implementation of $I-V$ curve non-ideality factor equal to 2. However, calculation [4] did not take into account Shockley-Read-Hall recombination current in the neutral part of base region. And there is no connection between the saturation current $I_{0 r}$ in [4] and such physical parameters as Shockley-Read-Hall recombination time $\tau_{S R}$, the doping level of semiconductor, the effective densities of states in the conduction and valence bands as well as the bandgap. The model [4] does not allow to calculate the $I_{0 r}$ value for particular semiconductor and regarded structure parameters. It denies SC optimization by the recombination current minimization.

The extended and modified GaAs SCs efficiency analysis in this paper uses some results of [5]. In particular, this research is correct for the case of an arbitrary injection level, i.e. for an arbitrary excess (generated by the light) concentration $\Delta p$ of electronhole pairs. The luminescence internal quantum yield for arbitrary $\Delta p$ is analyzed regarding main recombination mechanisms. The SCR recombination velocity and current corrected equations are determined and used to calculate the open-circuit voltage. The radiative recombination coefficient $A$ is calculated using Roosbroeck and Shockley equations [6]. This work introduces self-consistent analysis of factors influencing the photoconversion efficiency in more general assumptions than those in $[1,2,4]$.

In our work, the equation for the open-circuit voltage is written regarding the neutral part of the base region and SCR recombination currents. Saturation currents take into account radiative and nonradiative recombination and other semiconductor parameters, so the specific value of this ratio can lead to both the increase and decrease in this current. It is shown that this dependence in some cases is essential for the SC photovoltage calculation.

It is shown that the main SC parameters could not be chosen arbitrarily, because they are interdependent. For example, determining the doping level, it is necessary to consider the change in the light absorption coefficient and radiative recombination coefficient. It was found that reabsorption and re-emission of light are not major factors for the GaAs SCs efficiency.

These results can be used to select optimal parameters for SCs produced using both gallium arsenide and other semiconductor materials of industrial technologies (including germanium and silicon solar cells) and to achieve a maximum photoconversion efficiency.

\section{Basic relations and the analysis of the influence of various factors on the GaAs photoconversion efficiency}

Let us analyze GaAs photoconversion efficiency regarding the doping level and conductivity type, ratio of the cross-sections for carriers captured by deep levels, radiative and nonradiative recombination probabilities ratios for the SCR and the neutral part of the base region.

\subsection{Influence of doping level and conductivity type}

Let us analyze the behavior of the light absorption coefficient $\alpha$ of GaAs as a function of photon energy $E_{p h}$, depending on the conductivity type and the doping 
level of semiconductor. These dependences $\alpha\left(E_{p h}\right)$ are shown in Fig. 1 [5]. Fig. 1 shows the increase of the fundamental absorption edge blurry with increasing the doping level in $p$-type GaAs (Urbach effect), i.e. absorption increase in the spectral range where the photon energy $E_{p h}$ is less than the bandgap $E_{g}\left(E_{p h}<E_{g}\right)$. At the same time, in $n$-type GaAs at typical for TSCs levels of doping, there is electron gas degeneracy and Burstein-Moss effect domination. As a result, the doping level increase shifts the absorbtion edge to the highenergy region. Thus, the short-circuit current for a given thickness $d$ decreases.

Using the known $\alpha\left(E_{p h}\right)$ dependence [5] and the formula [6]

$$
A=\frac{(k T)^{3}}{\pi^{2} c^{2} \hbar^{3} n_{i}^{2}} \int_{0}^{\infty} \frac{\varepsilon(u) \alpha(u) u^{2} d u}{e^{u}-1},
$$
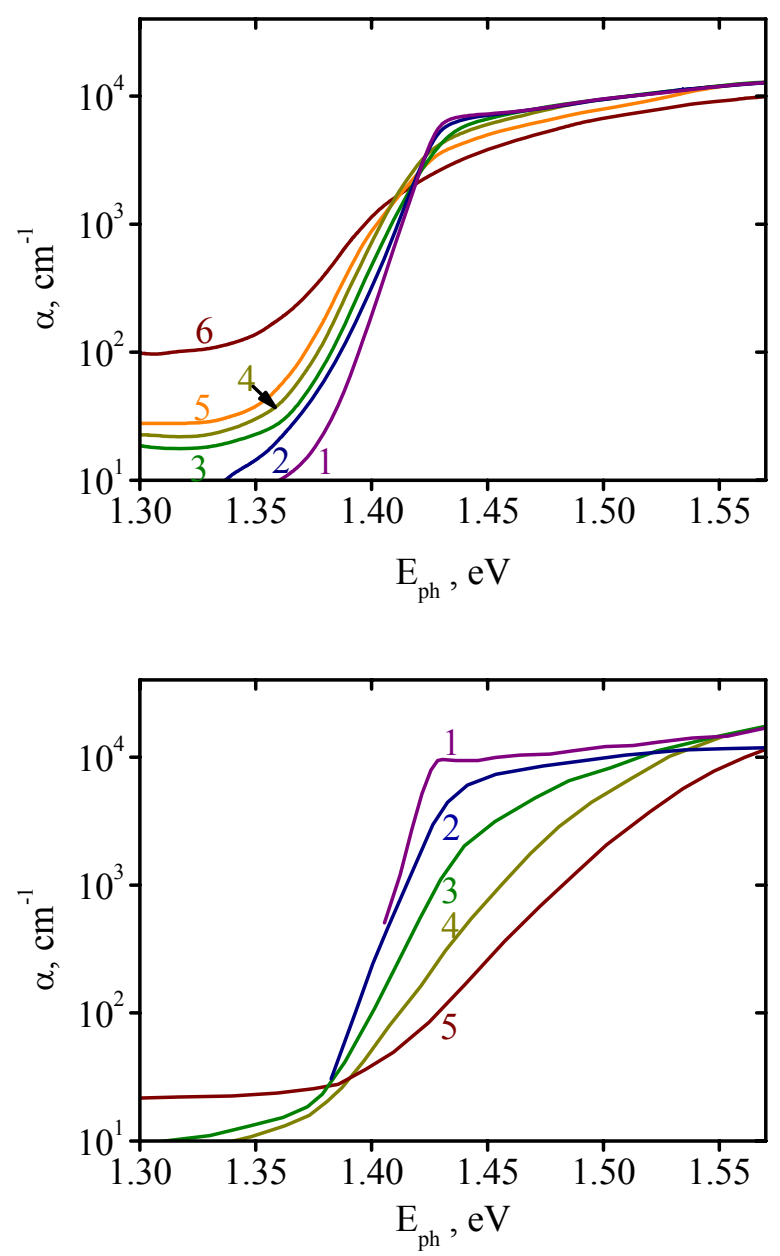

Fig. 1. Doping level influence on the absorption edge of $p$ - (a) and $n$-type (b) GaAs [5]. $p$-type doping level, $\mathrm{cm}^{-3}$ : $1.6 \cdot 10^{16}(1), 2.2 \cdot 10^{17}(2), 4.9 \cdot 10^{17}(3), 1.2 \cdot 10^{18}(4), 2.4 \cdot 10^{18}(5)$, $1.6 \cdot 10^{19}(6) ; n$-type doping level, $\mathrm{cm}^{-3}: 5 \cdot 10^{13}(1), 5.9 \cdot 10^{17}(2)$, $2 \cdot 10^{18}(3), 3.3 \cdot 10^{18}(4), 6.7 \cdot 10^{18}(5)$.

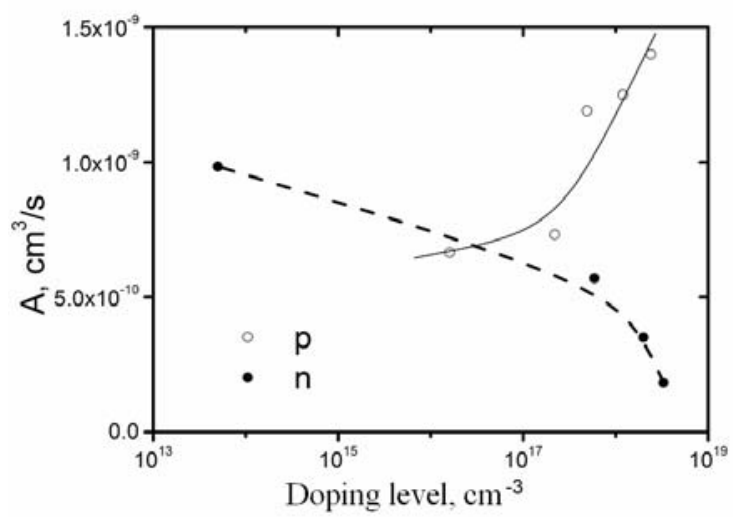

Fig. 2. The radiative recombination coefficient $A$ versus the doping level in $n$ - and $p$-type GaAs.

we can calculate the radiative recombination coefficient $A$. Here, $c$ is the speed of light, $\hbar$ - Planck's constant, $n_{i}-$ intrinsic concentration of semiconductor, $\varepsilon(u)-$ dielectric permittivity of semiconductor, taking into account the dispersion of the photon energy, $u=E_{p h} / k T$-dimensionless photon energy.

The calculated values of $A$ are shown in Fig. 2. The figure shows that in $p$-type semiconductors with the increasing doping level, the value $A$ increases from $7 \cdot 10^{-10} \mathrm{~cm}^{3} / \mathrm{s}$ for $p_{0}=10^{17} \mathrm{~cm}^{-3}$ up to $1.5 \cdot 10^{-9} \mathrm{~cm}^{3} / \mathrm{s}$ for $p_{0}=2 \cdot 10^{18} \mathrm{~cm}^{-3}$. At the same time, in $n$-type semiconductors with the increasing doping level, the value $A$ decreases from $10^{-9} \mathrm{~cm}^{3} / \mathrm{s}$ for $n_{0}=$ $10^{14} \mathrm{~cm}^{-3}$ down to $9 \cdot 10^{-11} \mathrm{~cm}^{3} / \mathrm{s}$ for $n_{0}=$ $2 \cdot 10^{18} \mathrm{~cm}^{-3}$.

The influence of the doping level on the value of bulk lifetime in GaAs [8-10] is shown in Fig. 3. In this case,

$\tau_{b}=\left(\frac{1}{\tau_{S R}}+\frac{1}{\tau_{r}}\right)^{-1}$,

where $\tau_{S R}$ is the Shockley-Read-Hall lifetime, $\tau_{r}=\left(A\left(N_{d}\right) N_{d}\right)^{-1}-$ radiative lifetime and $N_{d}$ - doping level of the base. Fig. 3 shows that the bulk lifetime in $p$ type GaAs is lower than that in $n$-type. Data of Fig. 3 correlate with the data on the lifetimes for $n$ - and $p$-type GaAs [9].

The contributions of $\tau_{r}$ and $\tau_{S R}$ can be separated using the known values of $A\left(N_{d}\right)$ and the relation (2). Using this way, the values of $\tau_{S R}$ for $n$ - and p-type GaAs with different doping levels can be achieved. The typical $\tau_{S R}$ value is equal to $2 \cdot 10^{-8} \mathrm{~s}$ for $n$-type GaAs in the range of doping levels from $1 \cdot 10^{16} \mathrm{~cm}^{-3}$ up to $5 \cdot 10^{18} \mathrm{~cm}^{-3}$. For the doping level $n_{0}$ of $10^{16} \mathrm{~cm}^{-3}$, the 
radiative lifetime $\tau_{r}$ is equal to $10^{-7} \mathrm{~s}$, while for $n_{0}=$ $2 \cdot 10^{18} \mathrm{~cm}^{-3}-\tau_{r}=2.5 \cdot 10^{-8} \mathrm{~s}$. In the first case, $\tau_{S R}$ is less than $\tau_{r}$, while in the second case the times $\tau_{S R}$ and $\tau_{r}$ are of the same order, i.e. radiative recombination does not dominate. For this case, photon recycling will be weakened even in highly reflective structures. Moreover, regarding to the photon recycling [4], the value of radiative recombination coefficient decreases. In this case, an effective radiative recombination coefficient $A_{\text {eff }}=A\left(1-\gamma_{r}\right)$ (here $\gamma_{r}$ is the photon recycling factor) should be used in the open-circuit voltage $V_{O C}$ calculation. This effect leads to the increase in the radiative lifetime and to the raise of nonradiative recombination contribution.

Typical $\tau_{S R}$ values in $p$-type semiconductors at low excitation levels $\left(\Delta p<<p_{0}\right)$ are about $5 \cdot 10^{-9} \mathrm{~s}$ $[8,9]$. At sufficiently high levels of excitation, $\tau_{S R}$ is about $2.5 \cdot 10^{-7} \mathrm{~s}$. Simple calculation allows using these values to determine the ratio of capture cross-sections for electrons and holes by recombination level, which in this case is equal to fifty.

Thus, as it follows from the analysis of known data about Shockley-Read-Hall lifetimes $\tau_{S R}$, for typical $\tau_{S R}$ values in $n$ - and $p$-type GaAs, $n$-type GaAs with higher $\tau_{S R}$ suites as the base material better. This doping type is also proposed in the Kayes patent [10].

\subsection{Internal quantum efficiency of the short-circuit current}

A more general expression for the quantum yield of the short-circuit current $q_{s c}\left(E_{p h}\right)$ [11] can be written as

$q_{S C}=q_{s p}+q_{s n}$ emitter, $\tau_{p}$ - bulk lifetime in the emitter, $R_{d}-$ light reflection coefficient of the back surface, $L=\left(D \tau_{b}\right)^{1 / 2}$ - diffusion length in the base, $D$ - diffusion coefficient in the base, $S_{0}$ - effective surface recombination velocity at the surface of the emitter, $S_{d}$ - effective surface recombination velocity at the back surface.

When the $L>>d$ criterion fulfils, and when $S_{0}$ and $S_{d}$ are small (compared to the recombination rate in the base volume), the expression for $q_{S}\left(E_{g}, E_{p h}\right)$ reduces to $1-\exp (-\alpha d)$ expression for $R_{d} \rightarrow 0$ (poorly reflecting structures of the type A (Fig. 4)), and to expression $1-\exp (-2 \alpha d)$ for $R_{d} \rightarrow 1$ (highly reflective structures of the type B (Fig. 4) [4]). However, for sufficiently low recombination times, when $L \leq d$, as well as for large values of $S_{0}$ and $S_{d}$ the short-circuit current quantum yield can be significantly reduced relative to the values above.

\subsection{The open-circuit voltage}

We obtained a more definite, than the commonly used, expression for the open-circuit voltage $V_{O C}$ of solar cell with the $p-n$ junction. We suppose that in this expression the recombination current consists of two components: the neutral region recombination current and the base region SCR recombination current. Here we assume that Shockley-Read-Hall recombination goes through the discrete level close enough to the middle of the bandgap. Expressions for current recombination in SCR ([12] p. 104) can not be used directly for the illuminated SC. Furthermore, they are obtained in assumption of the equality of recombination center capture cross-sections of electrons and holes. We determine the contribution of SCR recombination in the open-circuit voltage $V_{O C}$ and in the internal quantum

$$
\begin{aligned}
& q_{s p}=\frac{\alpha L_{p}}{\left(\alpha L_{p}\right)^{2}-1} \cdot \frac{\alpha L_{p}+S_{0} \frac{\tau_{p}}{L_{p}}\left(1-e^{-\alpha d_{p}}\right) \cosh \left(\frac{d_{p}}{L_{p}}\right)-e^{-\alpha d_{p}} \sinh \left(\frac{d_{p}}{L_{p}}\right)-\alpha L_{p} e^{-\alpha d_{p}}}{S_{0} \frac{\tau_{p}}{L_{p}} \sinh \left(\frac{d_{p}}{L_{p}}\right)+\cosh \left(\frac{d_{p}}{L_{p}}\right)}, \\
& q_{s n}=\frac{\alpha L e^{-\alpha d_{p}}}{1-(\alpha L)^{2}} \cdot\left\{\frac{\left[S_{d} \cosh \left(\frac{d}{L}\right)+\frac{D}{L} \sinh \left(\frac{d}{L}\right)\right]\left(1+R_{d} e^{-2 \alpha d}\right)+\left(\alpha D\left(1-R_{d}\right)-S_{d}\left(1+R_{d}\right)\right) e^{-\alpha d}-}{S_{d} \sinh \left(\frac{d}{L}\right)+\frac{D}{L} \cosh \left(\frac{d}{L}\right)}\right. \\
& \left.\frac{\left.-\alpha L S_{d}\left[\sinh \left(\frac{d}{L}\right)+\frac{D}{L} \cosh \left(\frac{d}{L}\right)\right]\left(1-R_{d} e^{-2 \alpha d}\right)\right]}{S_{d} \sinh \left(\frac{d}{L}\right)+\frac{D}{L} \cosh \left(\frac{d}{L}\right)}\right]
\end{aligned}
$$

Here, $\alpha$ is the light absorption coefficient, $L_{p}$ diffusion length in the emitter, $d_{p}-$ thickness of the yield of luminescence $q_{p l}$ more correctly for a realistic case when cross-sections are not equal. At first, we 


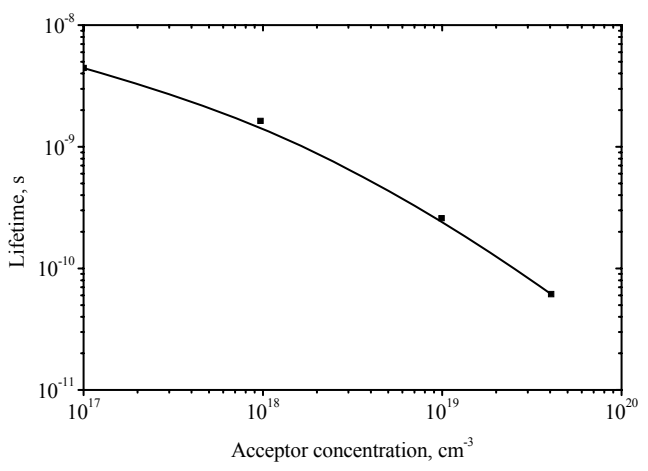

a)

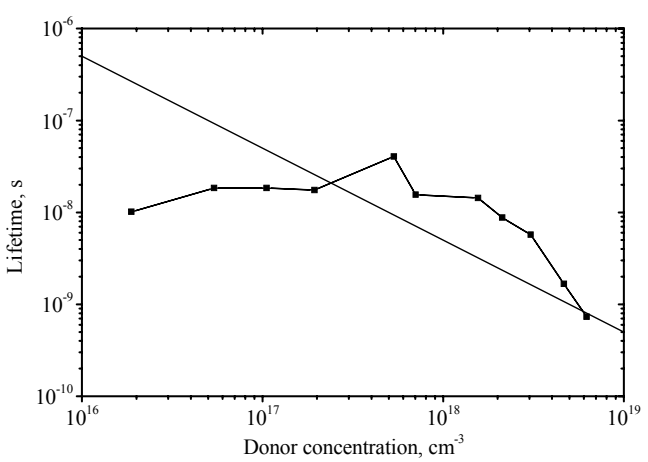

b)

Fig. 3. Bulk lifetime in $p$ - and $n$-type GaAs versus doping level [5]. Points are experimental values. Line in the figure for the $n$-type corresponds to the constant radiative recombination lifetime value.

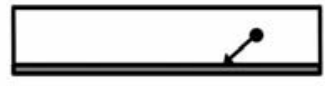

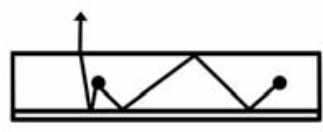

Fig. 4. Schematic diagrams of two types of planar solar cell structures, labeled Structures A and B. Structure A is a semiconductor slab with an absorbing substrate, Structure B is a semiconductor slab with a reflecting substrate. Source: [4].

obtain the value of the recombination velocity in SCR $V_{S C}$ for the illuminated $\mathrm{SC}$, when recombination in SCR goes through a deep recombination level. We assume the concentration of recombination centers $N_{r}$, the capture cross-section of electrons $\sigma_{n}$ and holes $\sigma_{p}$, energy placement is taken relative to the midgap. For $n$-type semiconductor under illumination, we can write

Here, $L_{D}=\left(\varepsilon_{0} \varepsilon_{S} k T / 2 q^{2} n_{0}\right)^{1 / 2}$ is the Debye
Boltzmann constant, $T$ - temperature, $q$ - elementary charge, $\Delta p$ - minority carriers excess concentration in the base, $\tau_{S R}=\left(C_{p} N_{r}\right)^{-1}-$ Shockley-Read-Hall lifetime, $C_{p}=V_{T} \sigma_{p}, C_{n}=V_{T} \sigma_{n}, b=\sigma_{p} / \sigma_{n}-$ carrier cross-sections ratio, $V_{T}-$ carrier average thermal velocity, $y_{p n}$ - the dimensionless potential at the $p-n$ junction interface, $y$ - the actual dimensionless (normalized to $k T / q$ ) electrostatic potential,

$n_{i}(T)=\sqrt{N_{c} N_{v}}\left(\frac{T}{300}\right)^{3 / 2} \exp \left(-\frac{E_{g}}{2 k T}\right)$

- intrinsic carriers concentration, $N_{c}$ and $N_{v}$ are effective densities of states in the conduction band and valence band for $T=300 \mathrm{~K} ; E_{g}$ is the bandgap and $\varepsilon_{r}=E_{r} / k T$.

The same deep recombination center responsible for recombination in SCR and for Shockley-Read-Hall recombination in the neutral base region of $\mathrm{SC}$ is assumed in (6) for simplicity.

$V_{S C}(\Delta p)$ calculation shows that for $n_{0} \approx 10^{7} \mathrm{~cm}^{-3}$ and $\Delta p$ ranged from $10^{12} \mathrm{~cm}^{-3}$ up to $10^{14} \mathrm{~cm}^{-3}$, corresponding to the $\mathrm{SC}$ operation conditions for different Shockley-Read-Hall recombination times, the recombination rate does not depend on the recombination energy position relative to the middle of bandgap $E_{r}$ value up to $\pm 0.2 \mathrm{eV}$. Thus, when the $E_{r}$ magnitude is less than $0.2 \mathrm{eV}$, the terms proportional to $n_{i}$ in the denominator of expression (6) integrand can be neglected. It means that under these conditions the expression (6) for the SCR recombination velocity $V_{S C}(\Delta p)$, written for deep recombination centers, located in the middle of the gap, is correct for the high enough separation (within $0.2 \mathrm{eV}$ ) of the recombination level from the middle of the bandgap.

Using the expression (6) for $E_{r}=0$ case, when subintegral function has a maximum and $y_{m}=\ln \left(n_{0} / b\left(p_{0}+\Delta p\right)\right) / 2$ SCR recombination velocity $V_{S C}$ can be written approximately as

$V_{S C}=\frac{\kappa L_{D}}{\tau_{S R}} \frac{\left(\frac{n_{0} / b}{\left(p_{0}+\Delta p\right)}\right)^{1 / 2}}{\sqrt{\frac{1}{2} \ln \left(\frac{n_{0} / b}{\left(p_{0}+\Delta p\right)}\right)}} \cdot$

Calculations (1), (3) show $\kappa$ increase from 1.9 to 2 with $V_{S C}(\Delta p)=\frac{L_{D}}{\tau_{S R}} \int_{y_{p n}}^{0} \frac{n_{0}}{\left[\left(n_{0} e^{y}+n_{i} e^{\varepsilon_{r}}\right)+b\left(p_{0}+\Delta p\right) e^{-y}+n_{i} e^{-\varepsilon_{r}}\right] \sqrt{-y+\left(e^{y}-1\right)+\frac{\left(p_{0}+\Delta p\right)}{n_{0}} e^{-y}}} d y$.

screening length, $\varepsilon_{0}$ - dielectric constant of vacuum, $\varepsilon_{S}$ - relative permittivity of the semiconductor, $k$ -
$\Delta p$ increase from $10^{10}$ up to $10^{14} \mathrm{~cm}^{-3}$. In this case, the 
calculation results by using the exact formula and approximate evaluation coincide with less than $5 \%$ error.

Fig. 5 shows the calculated $V_{S C}\left(E_{g}\right)$ dependences constructed using the formula (8). The parameter is the concentration $\Delta p$ of minority holes in the base. One can see from the figure that starting from $10^{10} \mathrm{~cm}^{-3} V_{S C}$ is not dependent on $E_{g}$. The $V_{S C}$ value decreases with the $\Delta p$ increase.

When illumination makes $\Delta p>>p_{0}$, the relationship between $V_{S C}$ and recombination saturation current density $J_{r S}$ is described by the relation [13]

$$
\begin{aligned}
& V_{S C}=\frac{J_{r S}}{q}\left(\frac{\Delta p}{p_{0}}\right)^{1 / 2} \Delta p^{-1} \\
& J_{r S} \approx \frac{q \kappa L_{D} b^{-1 / 2} n_{i}}{\tau_{S R} \sqrt{\frac{1}{2 b} \ln \left(\frac{q n_{0}\left(d / \tau_{b}+S_{S}\right)}{J_{g}}\right)}},
\end{aligned}
$$

where $S_{S}=S_{0}+S_{d}$.

The generation-recombination balance equation ignoring the SCR recombination

$$
J_{S C} \approx q\left(\frac{d}{\tau_{b}}+S_{S}\right) \Delta p
$$

was used in (10). The logarithm parameter in (10) is much greater than 1, so the first approximation can be used. Here, $J_{S C}$ is the short-circuit photocurrent density. The equation (11) is correct, when the diffusion length $L$ is greater than the base thickness $d$.

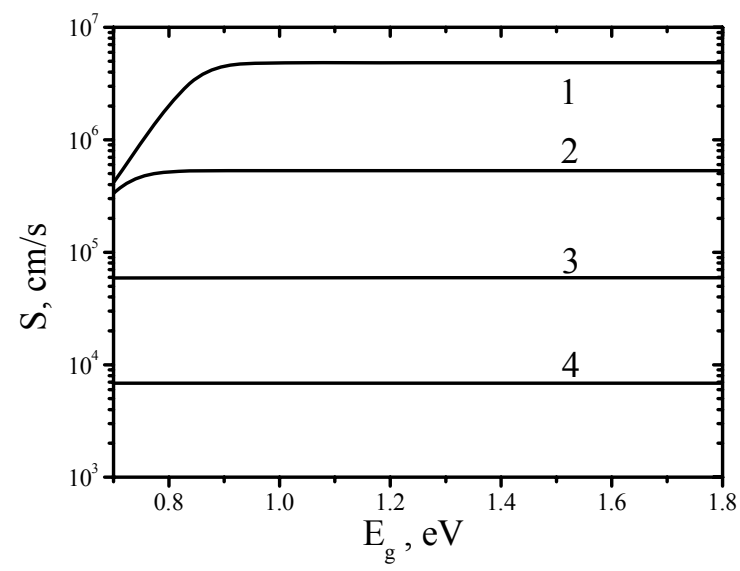

Fig. 5. Recombination velocity in SCR versus the bandgap for the following parameters: $\tau_{\mathrm{SR}}=10^{-7} \mathrm{~s}, \quad n_{0}=10^{17} \mathrm{~cm}^{-3}, T=$ $300 \mathrm{~K}, b=10^{-2} \mathrm{~s}, \Delta \mathrm{p}, \mathrm{cm}^{-3}: 1-10^{7} ; 2-10^{9} ; 3-10^{11} ; 4-10^{13}$.

Taking into account SCR recombination, the open circuit voltage $V_{O C}$ can be found via

$$
\begin{aligned}
& J_{g}=q\left(\frac{d}{\tau_{b}}+S_{S}\right) \frac{n_{i}^{2}}{n_{0}}\left[\exp \left(\frac{q V_{O C}}{k T}\right)-1\right]+ \\
& +q \frac{\kappa L_{D} b^{-1 / 2} n_{i}}{\tau_{S R} \sqrt{\frac{1}{2} \ln \left(\frac{1}{b} \frac{q n_{0}\left(d / \tau_{b}+S_{S}\right)}{J_{g}}\right)}}\left[\exp \left(\frac{q V_{O C}}{2 k T}\right)-1\right]
\end{aligned}
$$

when the second term is not much greater than the first one. If the second term is much more greater than the first one,

$$
\begin{aligned}
& J_{g}=q\left(\frac{d}{\tau_{b}}+S_{S}\right) \frac{n_{i}^{2}}{n_{0}}\left[\exp \left(\frac{q V_{O C}}{k T}\right)-1\right]+ \\
& +q \frac{\kappa L_{D} b^{-1 / 2} n_{i}}{\tau_{S R} \sqrt{\ln \left(\frac{\tau_{S R}}{q \kappa} \frac{J_{g}}{L_{D} n_{i} \exp \left(\frac{q V_{O C}}{2 k T}\right)}\right)}\left[\exp \left(\frac{q V_{O C}}{2 k T}\right)-1\right]}
\end{aligned}
$$

should be used to find the open-circuit voltage $V_{O C}$.

\subsection{The photoluminescence internal quantum yield}

Let us write the expression for the photoluminescence internal quantum yield regarding radiative recombination, bulk Shockley-Read-Hall one, Auger, surface and SCR ones for arbitrary excitation level $\Delta p$. We consider $p$-type semiconductor in the calculation. We take into account both the Shockley-Read-Hall time and the surface recombination velocity dependence on the excitation value for $b \neq 1$. Let us consider such nonradiative recombination parameters dependences on the equilibrium majority carriers concentration $p_{0}$, minority carrier concentration $n_{0}$ and excitation level $\Delta p$ as

$$
\begin{aligned}
& \tau_{S R}=\tau_{0}\left(1+b_{b}^{-1} \frac{n_{0}+\Delta p}{p_{0}+\Delta p}\right)^{-1}, \\
& S=S_{0}\left(1+b_{S}^{-1} \frac{n_{0}+\Delta p}{p_{0}+\Delta p}\right)^{-1}, \\
& S_{S C}=S_{S C 0}\left(\frac{b_{b} n_{0}}{\Delta p}\right)^{1 / 2}, R_{\text {Auger }}=C\left(p_{0}+\Delta p\right)^{2} .
\end{aligned}
$$

Here, $b_{b}$ and $b_{S}$ are capture cross-sections relations for bulk and surface recombination levels, respectively, $R_{\text {Auger }}$ is the Auger recombination rate, $C$ - Auger recombination coefficient.

Thus, $\tau_{S R}$ and $S$ dependences on $\Delta p$ and other parameters of (13) are typical for the Shockley-ReadHall recombination. $S_{S C}$ dependence on $\Delta p$ and other parameters is determined by (8).

Then in the case when $L>d$ (as required for efficient photoconversion), the expression for the 
luminescence internal quantum efficiency can be written as

$$
q_{p l}=\frac{d / \tau_{r}}{d\left(\tau_{S R}^{-1}+\tau_{r}^{-1}\right)+S+S_{S C}+R_{\text {Auger }}} .
$$

Fig. 6 shows the calculated dependence of the luminescence internal quantum yield versus excitation level using the following parameters: $p_{0}=3 \cdot 10^{17} \mathrm{~cm}^{-3}$, $S_{0}=1 \cdot 10^{2} \mathrm{~cm} / \mathrm{s}, \quad b_{p}=b_{S}=0.02 \quad[9], \quad d=1 \mu \mathrm{m}, \quad C=$ $7 \cdot 10^{-30} \mathrm{~cm}^{6} / \mathrm{s}$ [3], $S_{S C 0}$ is calculated using (7). The following $\tau_{0}$ values were used for 1-4 curves, respectively: $5 \cdot 10^{-9}, 2 \cdot 10^{-8}, 5 \cdot 10^{-8}$ and $10^{-6}$ s. Fig. 6 shows the luminescence internal quantum yield increase with the increase in $\tau_{0}$ value. When $\tau_{0}=10^{-6} \mathrm{~s}, q_{p l}$ value at its maximum is equal to $99.4 \%$, which is very close to the value obtained in [3].

However, the $\Delta p$ value in the SC under AM1.5 illumination is significantly lower. $\Delta p$ is determined by the generation-recombination balance equation taking into account all recombination components:

$J_{S C} / q=\left[d\left(\tau_{S R}^{-1}+\tau_{r}^{-1}\right)+S+S_{S C}+R_{\text {Auger }}\right] \Delta p$

The applied transcendent equations were solved numerically.

Fig. 7 shows $\Delta p$ dependence on the ShockleyRead-Hall lifetime for $A$ values of $5 \cdot 10^{-10} \mathrm{~cm}^{3} / \mathrm{s}$ and $10^{-11} \mathrm{~cm}^{3} / \mathrm{s}$. It can be seen that the curves for different $A$ values coincide, when $\tau_{S R} \leq 10^{-8} \mathrm{~s}$. The reason for this coincidence is in that $\Delta p \propto \tau_{S R}$ for this $\tau_{S R}$, i.e. $\Delta p$ is determined by the Shockley-Read-Hall recombination and does not depend on the radiative recombination. For these $\tau_{S R}$ values, the excitation level $\Delta p$ increases linearly with $\tau_{S R}$ increase, and for $\tau_{S R}$ equal to $5 \cdot 10^{-9} \mathrm{~s}$ and $5 \cdot 10^{-8} \mathrm{~s} \Delta p$ equals to $2.9 \cdot 10^{11}$ and $2.9 \cdot 10^{12} \mathrm{~cm}^{-3}$, respectively. Correlating these values with $q_{p l}$ dependences shown in Fig. 6, we find that for $b=0.02$ and $\tau_{S R}=5 \cdot 10^{-9} \mathrm{~s}$ the luminescence internal quantum yield $q_{p l}$ in $p$-type semiconductor is about 7\%, and for $\tau_{S R}=2 \cdot 10^{-8} \mathrm{~s} \quad q_{p l}=8 \%$. For $b=50$, the luminescence internal quantum yield $q_{p l}$ rises up to $32 \%$ and $37 \%$, respectively. And only for $\tau_{S R}=10^{-6} \mathrm{~s}$ and $\Delta p \approx 1.3 \cdot 10^{14} \mathrm{~cm}^{-3}, q_{p l}$ value is close to $93 \%$. Thus, $q_{p l}$ value is close to $100 \%$ only at laser excitation levels (obtained in [18]), when $\Delta p \geq 10^{17} \mathrm{~cm}^{-3}$. This situation does not correspond to the excitation levels of illuminated SC.

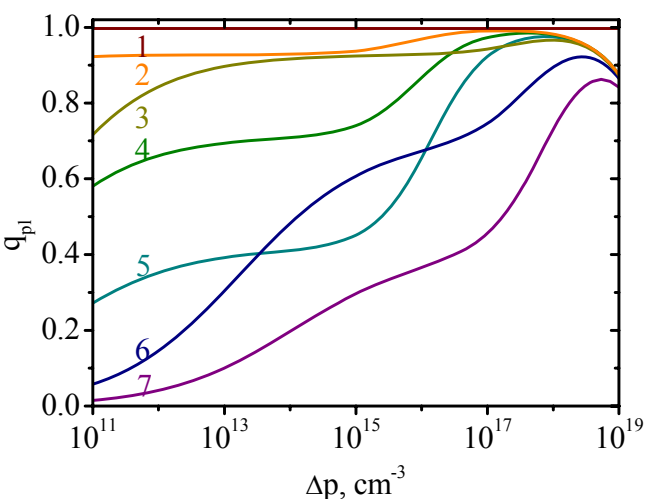

Fig. 6. Luminescence internal quantum yield versus the injection level. The used parameters: $p_{0}=3 \cdot 10^{17} \mathrm{~cm}^{-3}, d=$ $10^{-4} \mathrm{~cm}, A=5 \cdot 10^{-10} \mathrm{~cm}^{3} / \mathrm{s}, S=10^{3} \mathrm{~cm} / \mathrm{s}, \quad l-\tau_{\mathrm{SR}}=10 \mathrm{~s}$, $b=0.02 ; 2-\tau_{\mathrm{SR}}=10^{-6} \mathrm{~s}, b=0.02 ; 3-\tau_{\mathrm{SR}}=10^{-6} \mathrm{~s}, b=50$; $4-\tau_{\mathrm{SR}}=2 \cdot 10^{-8} \mathrm{~s}, b=0.02 ; 5-\tau_{\mathrm{SR}}=2 \cdot 10^{-8} \mathrm{~s}, b=50 ; 6-$ $\tau_{\mathrm{SR}}=5 \cdot 10^{-9} \mathrm{~s}, b=0.02 ; 7-\tau_{\mathrm{SR}}=5 \cdot 10^{-9} \mathrm{~s}, b=50$.

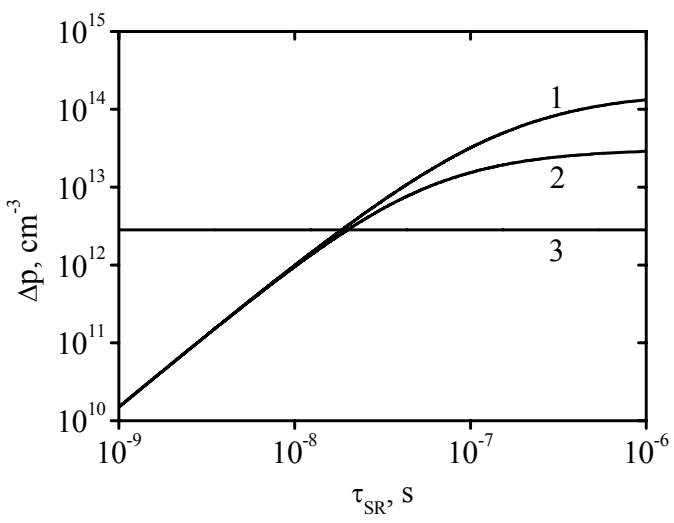

Fig. 7. Excitation level versus the Shockley-Read-Hall recombination lifetime. The used parameters: $p_{0}=10^{17} \mathrm{~cm}^{-3}$, $d=10^{-4} \mathrm{~cm}, S=10^{3} \mathrm{~cm} / \mathrm{s}, b=0.02, J_{S C}=30 \mathrm{~mA} / \mathrm{cm}^{2}, 1-A$, $10^{-11} \mathrm{~cm}^{3} / \mathrm{s}, 2-A=5 \cdot 10^{-10} \mathrm{~cm}^{3} / \mathrm{s}, 3-A=5 \cdot 10^{-10} \mathrm{~cm}^{3} / \mathrm{s}, \tau_{\mathrm{SR}}=$ $2 \cdot 10^{-8} \mathrm{~s}$.

Note that in the estimates above, the absence of the surface recombination influence on the luminescence internal quantum yield was assumed. However, GaAsAlGaAs system was used $[3,14]$ in the fabrication of structures with a high back surface reflectance, and the heterointerface recombination velocity lies within the range of $10^{2}-10^{4} \mathrm{~cm} / \mathrm{s}[15,16]$. For $\tau_{b}=\tau_{r}=10^{-7} \mathrm{~s}$, $d=10^{-4} \mathrm{~cm}, S_{S}=10^{3} \mathrm{~cm} / \mathrm{s}$, the luminescence internal quantum yield $q_{p l}$ is $33 \%$ (14). For $S_{S}>10^{3} \mathrm{~cm} / \mathrm{s}$, for example, when $S_{S}=10^{4} \mathrm{~cm} / \mathrm{s}$, we get even less $q_{p l}$ value. In the latter case, even in the absence of bulk ShockleyRead-Hall recombination, the luminescence quantum yield is essentially reduced due to the GaAs-AlGaAs heterojunction recombination. Note that GaAs-AlGaAs system is the most matched and has the smallest lattice constants mismatch. All other heterostructures have greater lattice mismatch and therefore greater $S_{S}$ values.

For $p-n$ junction GaAs solar cells, the typical $S_{0}$ and $S_{d}$ values are of the order of at least $10^{5} \mathrm{~cm} / \mathrm{s}$ [15]. The luminescence internal quantum yield $q_{p l}$ for such SCs is significantly less than 1 , so the effects of light re- 
emission and reabsorption (photon recycling) can be neglected even for large $\tau_{S R}$ values.

\subsection{The open-circuit voltage dependence on the semiconductor doping level}

Using saturation currents [4] and considering the photon recycling, the maximum obtainable value of the opencircuit voltage in direct-gap semiconductors $V_{O C}$ can be calculated (12). The radiative recombination saturation current density $J_{0 r}=7 \cdot 10^{2} \mathrm{~A} / \mathrm{cm}^{2}$ for B-type structures according to [4] was used to calculate the effective radiative recombination parameter $A_{\text {eff }}$ from

$J_{0 r}=q A_{e f f} d N_{c} N_{v}$.

For $d=2 \cdot 10^{-4} \mathrm{~cm}$ in highly reflective thick type B structures, the radiative recombination parameter $A_{\text {eff }}$ is about $4 \cdot 10^{-12} \mathrm{~cm}^{3} / \mathrm{s}$, and for poorly reflecting type A structures (in which, according to [4] $J_{0 r} \approx 1 \cdot 10^{4} \mathrm{~A} / \mathrm{cm}^{2}$ ), this parameter is about $10^{-10} \mathrm{~cm}^{3} / \mathrm{s}$. Substitution of the first value to the formula (12) by using the parameters of semiconductor with the bandgap $1.4 \mathrm{eV}$ (as in [2]) neglecting nonradiative recombination gives the value $1.142 \mathrm{~V}$ for $V_{O C}$, which is only in fourth sign different from the $V_{O C}$ value of $1.145 \mathrm{~V}[1,2]$.
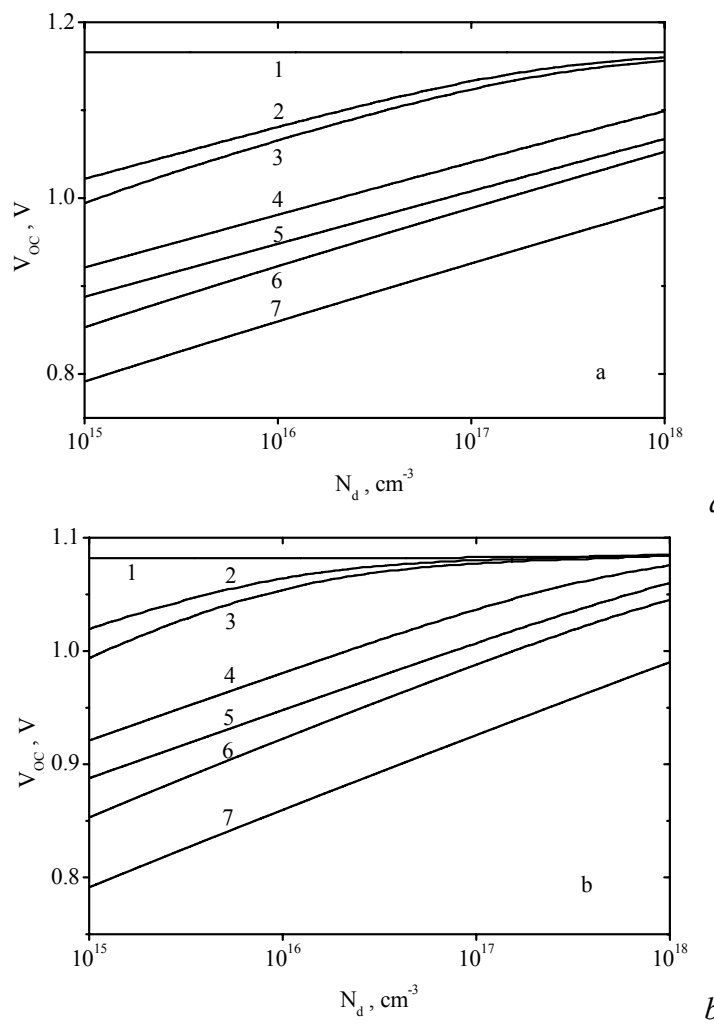

a)

Fig. 8. Open-circuit voltage dependence on the doping level. The used parameters: $d=10^{-4} \mathrm{~cm}, \varepsilon_{S}=12.8, S=1 \mathrm{~cm} / \mathrm{s}, T=300 \mathrm{~K}$, $E_{g}=1.42 \mathrm{eV}, D=10 \mathrm{~cm}^{2} / \mathrm{s}, S=1 \mathrm{~cm} / \mathrm{s}$. Fig. $8 \mathrm{a}$ is calculated for $A_{\text {eff }}=4 \cdot 10^{-12} \mathrm{~cm}^{3} / \mathrm{s}$, and Fig. $8 \mathrm{~b}$ is calculated for $A_{\text {eff }}=10^{-}$ ${ }^{10} \mathrm{~cm}^{3} / \mathrm{s}$. The curves parameters for Fig. 8: $1-\tau_{\mathrm{SR}}=10 \mathrm{~s}$, $b=0.02 ; 2-\tau_{\mathrm{SR}}=10^{-6} \mathrm{~s}, b=50 ; 3-\tau_{\mathrm{SR}}=10^{-6} \mathrm{~s}, b=0.02 ; 4-$ $\tau_{\mathrm{SR}}=2 \cdot 10^{-8} \mathrm{~s}, b=50 ; 5-\tau_{\mathrm{SR}}=2 \cdot 10^{-8} \mathrm{~s}, b=0.02 ; 6-\tau_{\mathrm{SR}}=$ $5 \cdot 10^{-9} \mathrm{~s}, b=50 ; 7-\tau_{\mathrm{SR}}=5 \cdot 10^{-9} \mathrm{~s}, b=0.02$.
The calculated open-circuit voltage $V_{O C}$ dependence for the type $\mathrm{A}$ and type $\mathrm{B}$ structures in the cases when $\tau_{S R}=2 \cdot 10^{-8} \mathrm{~s}$ (curves 1 and 2 ), $\tau_{S R}=$ $10^{-6} \mathrm{~s}$ (curves 3 and 4 ) and $\tau_{S R}=\infty$ (curves 5 and 6 ) on the doping level is plotted in Fig. 8. $A_{\text {eff }}$ of $10^{-10} \mathrm{~cm}^{3} / \mathrm{s}$ for the type A structures and $A_{\text {eff }}$ of $4 \cdot 10^{-12} \mathrm{~cm}^{3} / \mathrm{s}$ for the type B structures were used for Fig. 8 instead of $A$. The coincidence of $V_{O C}\left(n_{0}\right)$ dependences up to $n_{0}=10^{17} \mathrm{~cm}^{-3}$ for $\tau_{S R}=2 \cdot 10^{-8} \mathrm{~s}$ with the calculated $A_{\text {eff }}$ values means that in this doping levels area $V_{O C}\left(n_{0}\right)$ values are determined by nonradiative processes and are independent from the photon recycling. This dependence appears for $n_{0}>10^{17} \mathrm{~cm}^{-3}$, but the $V_{O C}\left(n_{0}\right)$ value continues to grow fast enough with the $n_{0}$ increase.

The $V_{O C}\left(n_{0}\right)$ values differ markedly among themselves for $\tau_{S R}=10^{-6} \mathrm{~s}$ starting with the doping levels $n_{0}$ of $10^{16} \mathrm{~cm}^{-3}$, but $V_{O C}$ value growth with $n_{0}$ increase continues. The figure shows that $V_{O C}\left(n_{0}\right)$ dependence is growing up to $n_{0}=10^{18} \mathrm{~cm}^{-3}$ except of the type A structures with $\tau_{S R}=10^{-6} \mathrm{~s}$. The $V_{O C}\left(n_{0}\right)$ dependence is stronger than the $V_{O C}\left(A_{\text {eff }}\right)$ dependence of Fig. 9 up to currently maximum Shockley-Read-Hall lifetimes of $10^{-6} \mathrm{~s}$. For example, the $V_{O C}\left(A_{\text {eff }}\right)$ value growth for $\tau_{S R}=2 \cdot 10^{-8} \mathrm{~s}$ is equal to $1.5 \%$, while the $V_{O C}\left(n_{0}\right)$ value growth is of $6 \%$. Even for $\tau_{S R}=10^{-6} \mathrm{~s}$, the $V_{O C}\left(A_{e f f}\right)$ value growth is of $8 \%$, and the $V_{O C}\left(n_{0}\right)$ value growth is of $11 \%$, i.e. $V_{O C}$ growth with $n_{0}$ prevails. It should be noted that nonradiative recombination dominates for $V_{S C}>d / \tau_{r}$, i.e. even for the cases when Shockley-Read-Hall lifetime is not less than the radiative lifetime $\left(\tau_{\mathrm{SR}} \geq \tau_{\mathrm{r}}\right)$. For example, for $\tau_{\mathrm{SR}}=\tau_{\mathrm{r}}=$ $10^{-7} \mathrm{~s}$ and $d=1 \mu \mathrm{m}$ the $V_{S C}$ value exceeds $d / \tau_{r}$ value by six times.

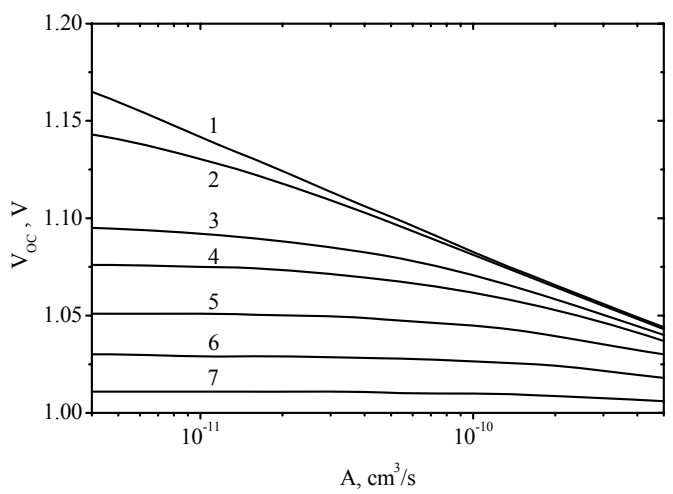

Fig. 9. Open-circuit voltage $V_{O C}$ dependence on the radiative recombination coefficient $A$ with account of nonradiative recombination. The used parameters: $n_{0}=2 \cdot 10^{17} \mathrm{~cm}^{-3}$, $d=2 \cdot 10^{-4} \mathrm{~cm}, T=300 \mathrm{~K}, E_{g}=1.42 \mathrm{eV}, D=10 \mathrm{~cm}^{2} / \mathrm{s}, S=$ $1 \mathrm{~cm} / \mathrm{s}, \varepsilon_{S}=12.8, b=1$.

\section{(C) 2014, V. Lashkaryov Institute of Semiconductor Physics, National Academy of Sciences of Ukraine}


In conclusion, we note that the use of the $J_{0 r}=$ $10^{4} \mathrm{~A} / \mathrm{cm}^{2}$ value for poorly reflecting structures and $J_{0 r}=7 \cdot 10^{2} \mathrm{~A} / \mathrm{cm}^{2}$ value for highly reflective structures leads to an overestimation of the photon recycling influence on the open-circuit voltage $V_{O C}$ while radiative and nonradiative recombinations make comparable contributions. This is due to the use of $100 \%$ luminescence internal quantum yield in the light reemission and reabsorption (photon recycling) $[1,2,4]$.

If the luminescence internal quantum yield is reduced (for instance, from $100 \%$ down to $50 \%$ ), only 1 2 successive reabsorption and re-emission acts will take place in highly reflective structures [1] instead of 30-40 acts. Thus, the $J_{0 r}$ value increases reducing the difference between $A$ and $A_{\text {eff. }}$ As a result re-emission and reabsorption processes influence will be decreased even earlier than the above calculations show. So, these formalisms are correct for $V_{O C}$ calculation in the absence of nonradiative processes only. In intermediate cases of a comparable contribution of the radiative and nonradiative recombination to the open-circuit voltage $V_{O C}$, the $V_{O C}$ values are less than values of Figs 8 and 9 .

\subsection{Influence of the capture cross-sections for electrons and holes on the open-circuit voltage}

Since the recombination velocity in SCR $V_{S C}$ and, therefore, the saturation current density depend on the ratio $b$ for cross-sections of charge carriers captured by deep recombination levels, the open-circuit voltage $V_{O C}$ will also depend on this ratio. According to (10) and (12), for $b>1 V_{S C}$ is decreased (so $V_{O C}$ is increased) and for $b<1 V_{S C}$ is increased (so $V_{O C}$ is decreased) in $n$-type semiconductors. The situation is reversed in $p$-type semiconductor, i.e. when $b>1 V_{O C}$ is decreased and when $b<1 V_{O C}$ is increased. Table 1 shows the $n$ - and $p$-type gallium arsenide $V_{O C}$ values, calculated for various $b$ values. Table 1 shows that $V_{O C}$ values can be significantly adjusted by the $b$ value. Particularly, the $V_{O C}$ value in $n$-type GaAs for $b=0.02$ is smaller than the value in the $p$-type one even when the Shockley-ReadHall lifetime $\tau_{S R}$ in the $n$-type exceeds $\tau_{S R}$ value in the $p$-type one. Thus, the $b$ value should be considered in selection of the material for highly efficient solar cells.

Table 1. The influence of the ratio $b$ of the cross-sections for electrons and holes captured by the deep recombination levels on the open-circuit voltage $V_{O C}$ for different Shockley-Read-Hall lifetimes $\tau_{\mathrm{SR}}$.

\begin{tabular}{|c|c|c|}
\hline$b$ & $\tau_{\mathrm{SR}}, \mathrm{s}$ & $V_{O C}, \mathrm{~V}$ \\
\hline 0.02 & $5 \cdot 10^{-9}$ & 0.945 \\
\hline 0.02 & $8 \cdot 10^{-8}$ & 1.007 \\
\hline 50 & $5 \cdot 10^{-9}$ & 1.024 \\
\hline 50 & $2 \cdot 10^{-8}$ & 1.051 \\
\hline 0.02 & $10^{-6}$ & 1.080 \\
\hline 50 & $10^{-6}$ & 1.082 \\
\hline
\end{tabular}

Table 2. The radiative recombination parameter $A$ and short-circuit current density $J_{S C}$ as functions of acceptor concentration $N_{a}$ (p-type) and donor concentration $N_{d}(n-$ type) in GaAs.

\begin{tabular}{|c|l|l|}
\hline$N_{a}, \mathrm{~cm}^{-3}$ & $A, \mathrm{~cm}^{3} / \mathrm{s}$ & $J_{S C}, \mathrm{~mA} / \mathrm{cm}^{2}$ \\
\hline $1.6 \cdot 10^{16}$ & $6.65 \cdot 10^{-10}$ & 31.663 \\
\hline $2.2 \cdot 10^{17}$ & $7.32 \cdot 10^{-10}$ & 31.376 \\
\hline $4.9 \cdot 10^{17}$ & $11.9 \cdot 10^{-10}$ & 30.642 \\
\hline $1.2 \cdot 10^{18}$ & $12.5 \cdot 10^{-10}$ & 29.308 \\
\hline $2.4 \cdot 10^{18}$ & $14.0 \cdot 10^{-10}$ & 27.271 \\
\hline $5 \cdot 10^{13}$ & $9.84 \cdot 10^{-10}$ & 31.813 \\
\hline $5.9 \cdot 10^{17}$ & $5.69 \cdot 10^{-10}$ & 27.400 \\
\hline $2 \cdot 10^{18}$ & $3.51 \cdot 10^{-10}$ & 24.937 \\
\hline $3.3 \cdot 10^{18}$ & $1.82 \cdot 10^{-10}$ & 24.815 \\
\hline
\end{tabular}

\subsection{Influence of the doping type and level on the short- circuit current}

An analysis of the doping type and level influence on the short-circuit current magnitude is carried out on typical direct-gap semiconductor - gallium arsenide. Shortcircuit current density under AM1.5 conditions in the case of full absorption of the incident light, neglecting SC illuminated surface shading by electrodes, is defined by the relationship:

$$
J_{S C}=\left(1-R_{S}\right)(1-m) \int_{E_{g}}^{\infty} J_{A M 1.5}\left(E_{p h}\right) q_{S C}(\alpha) d E_{p h} .
$$

Here, $R_{S}$ is the light reflection coefficient on the SC illuminated surface, $m$ - illuminated surface shading by the contact grid factor, $J_{A M 1.5}$ - spectral photocurrent density for AM1.5 illumination.

Since the gallium arsenide light absorption coefficient depends essentially on both doping level and conductivity polarity, this dependence should be taken into account in the short-circuit current $J_{S C}$ calculation. The results of this calculation for gallium arsenide are shown in Table 2 . It shows that $J_{S C}$ value for $p$-type semiconductor increases with the doping level increase. This growth is associated with an increase in fundamental absorption edge blur with the doping level increase (see Fig. 1). At the same time, the $J_{S C}$ value for $n$-type semiconductor decreases with the increase in doping level due to the conduction band filling by carriers (Burstein-Moss effect).

\section{Direct-gap semiconductors photoconversion efficiency}

Photoconversion efficiency calculation was fulfilled for GaAs SC as an example. The equation for $I-$ $V$ curve was written as 


$$
\begin{aligned}
& J(V)=J_{S C}-q\left(\frac{d}{\tau_{b}}+S_{S}\right) \frac{n_{i}^{2}}{n_{0}}\left[\exp \left(\frac{q V}{k T}\right)-1\right]- \\
& -q \frac{\kappa L_{D} b^{-1 / 2} n_{i}}{\tau_{S R} \sqrt{\frac{1}{2} \ln \left(\frac{q n_{0}\left(d / \tau_{b}+S_{S}\right)}{J_{g} b}\right)}}\left[\exp \left(\frac{q V}{2 k T}\right)-1\right] .
\end{aligned}
$$

The photovoltage at maximum takeoff power $V_{m}$ can be found from $d\{V J(V)\} / d V=0$ condition. The photocurrent density $J_{m}$ for this case can be calculated by the substitution of $V_{m}$ into (18). Finally, the photoconversion efficiency can be written as

$$
\eta=\frac{J_{m} V_{m}}{P_{S}}
$$

where $P_{S}$ is the illumination power density per unit area.

Fig. 10 shows the calculated using (19) GaAs photoconversion efficiency values versus the base material doping level for both conductivity polarities and the following Shockley-Read-Hall lifetimes $\tau_{S R}: 5 \cdot 10^{-9} \mathrm{~s}$ for $p$-type and $2 \cdot 10^{-8} \mathrm{~s}$ for the $n$-type. Zero $R_{S}$ and $m$ values were applied.

One can see from the figure that photoconversion efficiency $\eta$ increases with the doping level growth for both $p$ - and $n$-type. This increase is first of all due to an increase of $V_{O C}$. At higher doping levels, $\eta$ decreases due to the interband Auger recombination predominance. Lower $n$-type $\mathrm{SC} \eta$ values in this region are associated with lower $J_{S C}$ values. Maximum $\eta$ values for both $p$ - and $n$-type conductivity are realized at doping levels of about $10^{17} \mathrm{~cm}^{-3}$ and are close to the value of about $27 \%$ for typical $\tau_{S R}$ values. Thus, this doping level is optimal for the maximum efficiency $\eta$ achievement in gallium arsenide.

Fig. 11 shows calculated $\eta$ values versus the $b$ value for $p$ - and $n$-type gallium arsenide SCs. It can be seen that the maximum $\eta$ values for $p$ - and $n$-type gallium arsenide SCs are very similar, but the minimum values are much smaller for $n$-type. It is obvious that $n$ type Shockley-Read-Hall lifetime is less than the $p$-type lifetime. It leads to a significant $V_{m}$ value decrease.

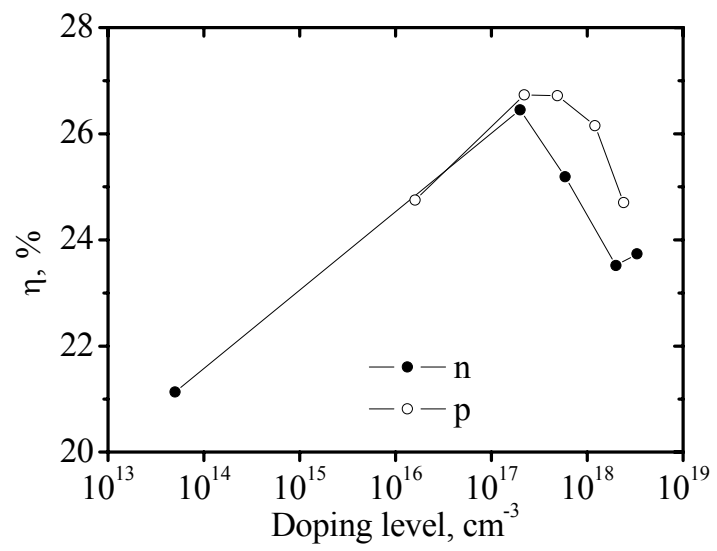

Fig. 10. Photoconversion efficiency dependence on the doping level.

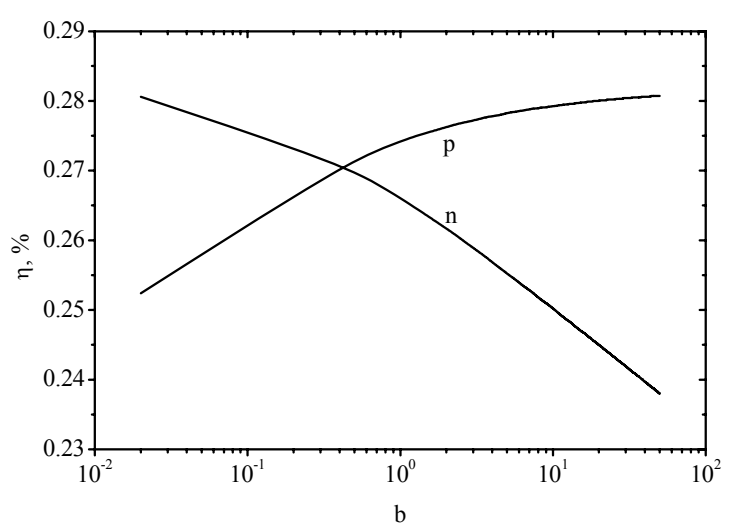

Fig. 11. Photoconversion efficiency versus the ratio $b$ for the capture cross-sections of electrons and holes in GaAs. The used parameters: $n_{0}\left(p_{0}\right)=2 \cdot 10^{17} \mathrm{~cm}^{-3}, A=5 \cdot 10^{-10} \mathrm{~cm}^{3} / \mathrm{s}, E_{g}=$ $1.42 \mathrm{eV}, d=10^{-4} \mathrm{~cm}, \varepsilon_{\mathrm{S}}=12.8, S=10^{3} \mathrm{~cm} / \mathrm{s} ; p$-type: $J_{S C}=$ $31.6 \mathrm{~mA} / \mathrm{cm}^{2}, \tau_{S R}=5 \cdot 10^{-9} \mathrm{~s}, D=50 \mathrm{~cm}^{2} / \mathrm{s} ; n$-type: $J_{S C}=$ $30.9 \mathrm{~mA} / \mathrm{cm}^{2}, \tau_{S R}=2 \cdot 10^{-8} \mathrm{~s}, D=10 \mathrm{~cm}^{2} / \mathrm{s}$.

Therefore, to achieve both high open-circuit voltage value and high short-circuit current density, i.e. to get the maximum photoconversion efficiency $\eta$, we must use $p$-type semiconductor as the base material. Note that the diffusion length of electrons in the $p$-type base material is much greater than the diffusion length of holes in the $n$-type base material. It is possible that the $p$ type GaAs disadvantage compared to the $n$-type is the lower Shockley-Read-Hall lifetime $\tau_{S R}$. However, according to $[7,8]$, typical $p$-type lifetimes $\tau_{S R}$ are not so small compared to the $n$-type, so it is prematurely to do the final conclusions about the $n$-type.

We also note that, even for the maximum ShockleyRead-Hall lifetime of $10^{-6} \mathrm{~s}$ achieved, the photon recycling neglection leads to no more than $8 \%$ reduction in the case of highly reflective structures, and to no more than $3 \%$ reduction in the case of poorly reflecting structures. These differences are estimated to be not so great compared to, for example, the summarized influence of the ratio of the surface recombination velocity and cross-sections for electrons and holes captured by the recombination levels, especially with the doping level influence on the $V_{O C}$ value. Therefore, the influence of these factors on the photoconversion efficiency must be considered in the first place.

\section{Photoconversion efficiency analysis features for other semiconductors (the indirect and direct band)}

The above relationships can be used to optimize the other direct-gap semiconductor solar cells (including $\mathrm{A}^{3} \mathrm{~B}^{5}$ group) parameters. First of all, the article includes general relations allowing to fulfil a specified analysis. Second, a number of these semiconductor parameters are very close to the GaAs parameters. In particular, the electrons and holes effective masses that determine the mobility and diffusion coefficients in these semiconductors are very close to the GaAs ones. 
Consequently, the densities of states in the conduction and the valence bands, as well as radiative recombination coefficients are close. The ShockleyRead-Hall lifetimes should be close as well. The single difference is the relation between the radiative and nonradiative processes in the semiconductor volume for these materials that changes in favor of the radiative processes dominance with the bandgap $E_{g}$ decrease. So, the photon recycling growth for narrow-gap semiconductors is possible. However, the following two conditions have to be performed. First, this situation is possible only for small surface recombination velocity values. But the fulfilment of this condition is very difficult. The AlGaAs-GaAs systems, as mentioned, have interfaces of very high quality, with the lowest recombination velocities. Heterojunction interfaces of other semiconductors are not perfect, and the recombination velocities at the boundaries are much higher than those for the AlGaAs-GaAs system. This fact eliminates the role of the radiative processes.

Second, it is difficult to realize good light reflection conditions in the each cell of multi-junction SC, because it needs a certain relationship between the refractive indices of the neighbor cells. This condition limits the photon recycling again. Finally, the open-circuit voltage growth for the photon recycling is inessential for the actual Shockley-Read-Hall recombination times. Regarding to the faster open-circuit voltage decrease than the bandgap decrease with bandgap narrowing, the error due to the neglect of photon recycling in the total open-circuit voltage value for the typical $\tau_{S R}$ values will be small.

\subsection{Indirect semiconductors (silicon)}

The obtained relations can be used in a number of cases to optimize the indirect semiconductor (for example, silicon, germanium) solar cells parameters, in which the radiative recombination probability is low. Silicon and germanium are particularly used as a third element in some three-junction SCs. It is especially important for monocrystalline and multicrystalline silicon solar cells, as silicon solar modules and batteries are widely used. Moreover, monocrystalline and multicrystalline silicon SCs produce the prevalent part of electricity generated by direct solar energy conversion up to date.

Until recently, high-efficiency silicon solar cell parameters were modeled using the numerical solution of the drift and diffusion equations $[9,12]$. There were also attempts $[4,17]$ to model silicon solar cells efficiency in the same approximations as gallium arsenide SCs, i.e. taking into account the photon recycling (re-emission and absorption of photons) in highly reflective structures or structures with multiple reflection (equal to the raise of the optical thickness). The photon recycling for silicon is assumed to be much lower than for gallium arsenide, where the luminescence internal quantum yield is assumed to be near $100 \%$ (as demonstrated for the high excitation case [3]). For example, the maximum photoluminescence external quantum yield of just $6.1 \%$ was obtained [18] at room temperature. To estimate the luminescence internal quantum yield $q_{p l}$ for silicon, we use the equation (14) with the following interband Auger recombination parameter $[11,19]$ :

$R_{\text {Auger }}=C_{p}\left(p_{0}+\Delta p\right)^{2}+C_{n}\left(p_{0}+\Delta p\right) \Delta p$,

where

$C_{p}=10^{-31} \mathrm{~cm}^{6} / \mathrm{s}$ and $C_{n}=\left(2.8 \cdot 10^{-31}+\frac{2.5 \cdot 10^{-22}}{\Delta p^{0.5}}\right) \mathrm{cm}^{6} / \mathrm{s}$. The second term in the expression for $C_{n}$ includes the spatial correlation due to Coulomb interaction effect in the distribution of two electrons and one hole needed to act Auger recombination.

The $q_{p l}(\Delta p)$ calculation results for the case of silicon are shown in Fig. 12. The value of $6 \cdot 10^{-15} \mathrm{~cm}^{3} / \mathrm{s}$ [20] was used as the radiative recombination parameter at $T=300 \mathrm{~K}$. It can be seen from the figure (see curve 1) that the highest value of $99.3 \%$ is realized at $\Delta p=$ $10^{10} \mathrm{~cm}^{-3}$ and $p_{0}=10^{14} \mathrm{~cm}^{-3}$ for the case of interband Auger recombination, as the only nonradiative recombination channel is present. In the case of minimal recombination parameters $\left(\tau_{S R}=38 \mathrm{~ms}, S=0.25 \mathrm{~cm} / \mathrm{s}\right)$ $[21,22]$ achieved in silicon, the largest $q_{p l}$ value is $52.7 \%$ (curve 2). For the silicon solar cell parameters, providing the record efficiency equal to $25 \%$ [23], the highest $q_{p l}$ value is $4 \%$ (curve 3). At $\Delta p \geq 10^{16} \mathrm{~cm}^{-3}$, there is a strong $q_{p l}$ drop associated with the increased nonradiative Auger recombination contribution. Since a typical $\Delta p$ value for a silicon solar cell under illumination is greater than $10^{16} \mathrm{~cm}^{-3}$, as it is shown by the calculation (15), $q_{p l}$ value for this situation does not exceed 25\%, as it is shown in Fig. 12. It means that the photon recycling can be neglected in the silicon solar cell parameters (including the maximum achievable one) simulation.

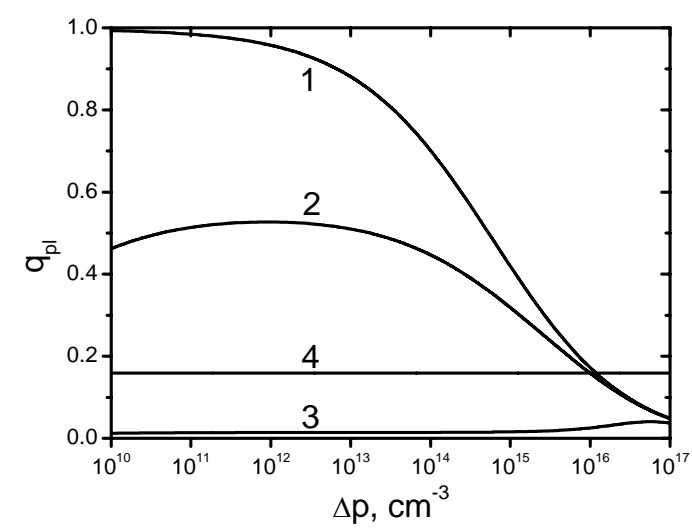

Fig. 12. Luminescence internal quantum yield dependence on the excitation level in silicon. The used parameters: $d=200 \mu \mathrm{m}$, $T=298 \mathrm{~K} ; 1-p_{0}=3 \cdot 10^{16} \mathrm{~cm}^{-3}, \tau_{S R}=10^{2} \mathrm{~s}, S=10^{-3} \mathrm{~cm} / \mathrm{s}$; $2-p_{0}=8 \cdot 10^{16} \mathrm{~cm}^{-3}, \quad \tau_{S R}=3.8 \cdot 10^{-2} \mathrm{~s}, \quad S=10 \mathrm{~cm} / \mathrm{s} ; \quad 3-$ $p_{0}=1 \cdot 10^{16} \mathrm{~cm}^{-3}, \tau_{S R}=10^{-3} \mathrm{~s}, S=60 \mathrm{~cm} / \mathrm{s}$. 
Note that the above formulae are not suitable for the silicon solar cell efficiency calculation in the case of over $20 \%$ efficiency. The matter is that these solar cells are manufactured from high quality monocrystalline silicon with the Shockley-Read-Hall lifetime not less than $1 \mathrm{~ms}$. Therefore, the diffusion length $L$ for such SCs can reach more than one millimeter, and this length substantially exceeds the base thickness $d$. The electronhole pairs excess concentration in the open-circuit mode, as it has been said above, reaches values of the order of $10^{16} \mathrm{~cm}^{-3}$. If the base doping is less than this value, the open-circuit voltage $V_{O C}$ in $p$-type semiconductor is determined by the following expression [11]

$V_{O C} \cong \frac{k T}{q} \ln \left(\frac{\Delta p}{n_{0}}\right)+\frac{k T}{q} \ln \left(1+\frac{\Delta p}{p_{0}}\right)$.

The greater $\Delta p / p_{0}$ ratio is in comparison to unity, the greater is the open-circuit voltage in a highly efficient silicon solar cell as compared to the case where $L<d$. This is associated not only with a higher $\Delta p$ value, but also with the noticeable or even comparable to the illuminated surface voltage value contribution of the back surface to open-circuit voltage. Such a situation is never realized in direct-gap semiconductors, like to gallium arsenide, due to quite a short non-equilibrium charge carrier lifetime and $\Delta p<<p_{0}$.

The $25 \%$ record photoconversion efficiency in silicon under AM1.5 conditions is set [23] especially for the case above where the formula (21) is correct. In addition to an almost complete incident light absorption by the SC implementation (that can be achieved through the use of geometric relief), the main problem is to minimize the surface recombination velocity values on the illuminated and back surfaces. The illuminated surface recombination velocity was minimized by thermal oxide with a low density of surface states growth and the back surface recombination velocity minimization was achieved by the isotype junction creation by Green [23]. There are recent researches solving this problem by $\alpha-\mathrm{Si}: \mathrm{H}-\mathrm{Si}$ heterojunctions usage $[24,25]$. In this case, dangling bonds passivation with hydrogen to minimize the surface recombination velocity $S$ is necessary. The highest $24.7 \%$ efficiency of these solar cells was obtained in [25]. It is necessary to determine the $\Delta p$ value from the equation (15) and substitute this $\Delta p$ in (21) to find the $V_{O C}$ value in this case.

Fig. 13 shows the $V_{O C}$ dependence on the base doping level. The curve 1 corresponds to the limiting parameters, when the Shockley-Read-Hall recombination velocity and the surface recombination velocity are negligibly small as compared to the interband Auger recombination one. The curve 2 corresponds to the case of actual silicon parameters ( $\tau_{S R}=38 \mathrm{~ms}, S=10 \mathrm{~cm} / \mathrm{s}$ ), and the curve 3 simulates the $V_{O C}$ value for the silicon solar cell with a record efficiency of $25 \%$ [23]. All the curves have the maxima that lie at $p_{0} \leq 10^{17} \mathrm{~cm}^{-3}$ as can be seen from the figure. $V_{O C}$ growth is associated with a relative decrease in the second term (21) contribution with the $p_{0}$ increase in the region of $p_{0}<10^{17} \mathrm{~cm}^{-3}$. $V_{O C}$ decrease is associated with $R_{\text {Auger }}$ growth for $p_{0} \geq 10^{17} \mathrm{~cm}^{-3}$. The calculated using (21) open-circuit voltage value can exceed $0.740 \mathrm{~V}$ for the record SC parameters. Conventional silicon solar cells with $L<d$ can have such a $V_{O C}$ value only under concentrated illumination.

The equation (21) is a quadratic equation as regards to $\Delta p$, and its solution has the following form:

$\Delta p=-\frac{p_{0}}{2}+\sqrt{\frac{p_{0}{ }^{2}}{4}+\exp \left(\frac{q V_{O C}}{k T}\right)}$.

Replacing $V_{O C}$ in (22) by the applied forward bias $V$, we get the $\Delta p$ to $V$ relation. It allows to write current-voltage characteristic based on the expression (15)

$J(V)=J_{S C}-J_{r e c}(V)$.

Further, we find $V_{m}$ from the maximum takeoff power condition $d(V J(V)) / d V=0$, and its substitution in (23) allows the $J_{m}$ value determining. As a result, we obtain the silicon solar cell photoconversion efficiency under AM 1.5 illumination:

$\eta=\frac{J_{m} V_{m}}{P_{S}}$

Fig. 14 shows the photoconversion efficiency dependence on the Shockley-Read-Hall lifetime $\tau_{S R}$. The basic parameters are the same as those in the previous figure. As one can see from the figure, for sufficiently large $\tau_{S R}$ values $\eta$ reaches its maximum and saturates. Saturation comes earlier for the larger $S$ values.

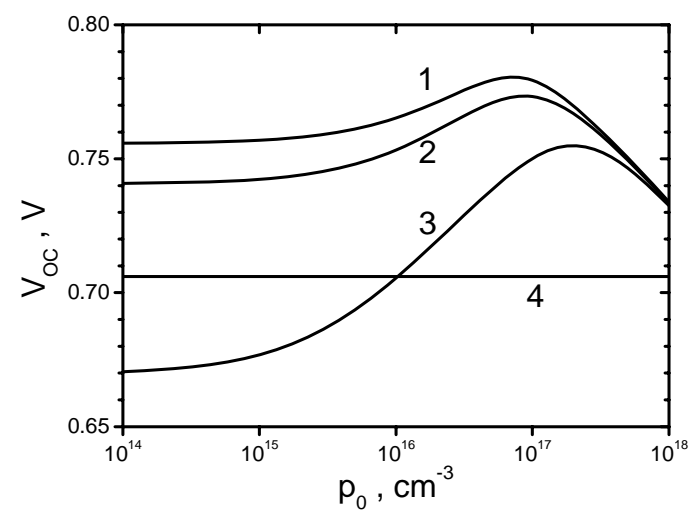

Fig. 13. Open-circuit voltage dependence on the doping level. The used parameters: $d=200 \mu \mathrm{m}, T=298 \mathrm{~K}$. The same $\tau_{S R}$ and $S$ values as for Fig. 12. The short-circuit current density $J_{S C}$ was assumed as: $1-43.5 \mathrm{~mA} / \mathrm{cm}^{2}, 2-42.7 \mathrm{~mA} / \mathrm{cm}^{2}, 3-$ $42.7 \mathrm{~mA} / \mathrm{cm}^{2}$.

\section{(C) 2014, V. Lashkaryov Institute of Semiconductor Physics, National Academy of Sciences of Ukraine}




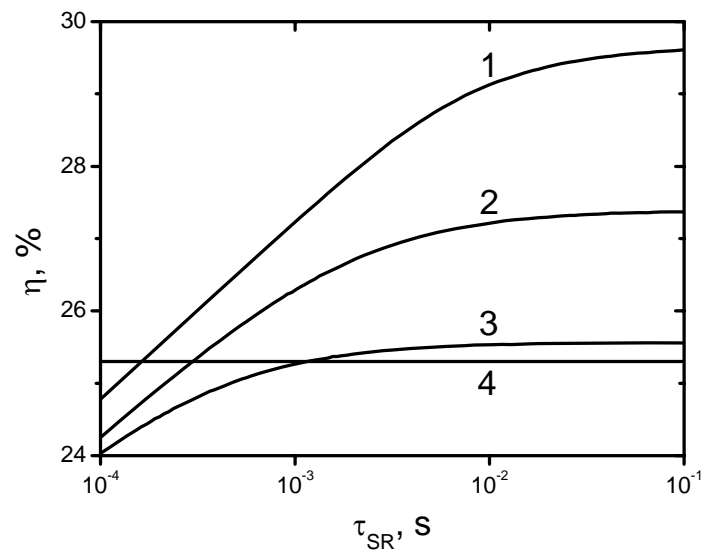

Fig. 14. Photoconversion efficiency $\eta$ versus the lifetime $\tau_{S R}$. The used parameters: $d=200 \mu \mathrm{m}, T=298 \mathrm{~K}$. The same $S$ values as for Fig. 13. The doping level was assumed to be $10^{16} \mathrm{~cm}^{-3}$.

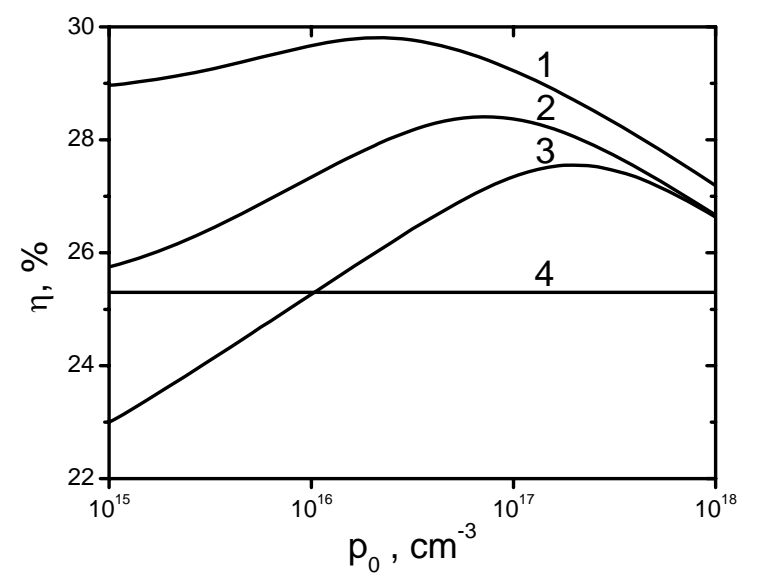

Fig. 15. Photoconversion efficiency $\eta$ versus the doping level. The used parameters: $d=200 \mu \mathrm{m}, T=298 \mathrm{~K}$. The same parameters as for Fig. 13.

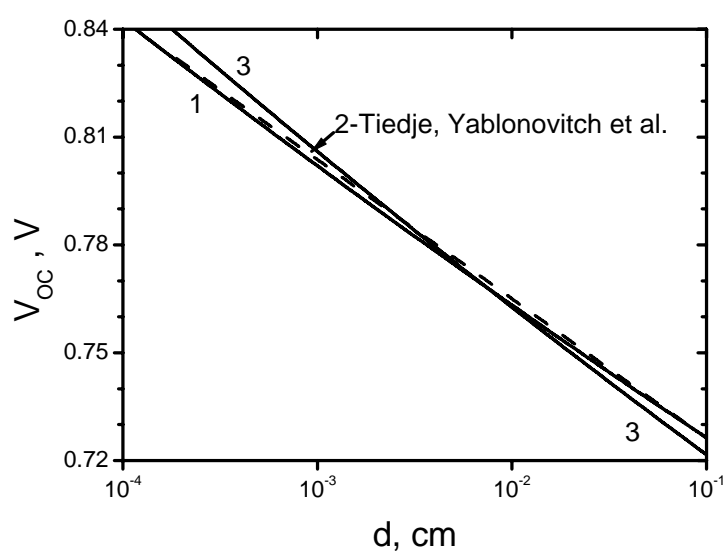

Fig. 16. Calculated open-circuit voltage versus the thickness of the limit silicon solar cell obtained in [17] (1), calculated using the parameters of [17] (2) and calculated using the parameters of our papers $[19,20](3)$.
Table 3. Experimental and calculated values of the main silicon solar cell parameters for three different samples [23-25].

\begin{tabular}{|c|c|c|c|c|c|}
\hline $\begin{array}{c}\text { Number } \\
\text { of cell }\end{array}$ & $\begin{array}{c}J_{S C}^{\exp }, \\
\mathrm{mA} / \mathrm{cm}^{2}\end{array}$ & $\begin{array}{c}V_{O C}^{\exp }, \\
\mathrm{V}\end{array}$ & $\begin{array}{c}V_{O C}^{\text {th }}, \\
\mathrm{V}\end{array}$ & $\begin{array}{c}\eta_{\exp }, \\
\%\end{array}$ & $\begin{array}{c}\eta_{\text {th, }} \\
\%\end{array}$ \\
\hline 1 & 427 & 0.706 & 0.706 & 25 & 25.3 \\
\hline 2 & 394 & 0.745 & 0.745 & 23.7 & 23.8 \\
\hline 3 & 395 & 0.750 & 0.750 & 24.7 & 24.9 \\
\hline
\end{tabular}

Fig. 15 shows the $\eta$ dependence on the base doping level $p_{0}$, which is calculated with the same basic parameters. It can be seen that all three curves have maxima. The lower $p_{0}$ values in maxima are realized for smaller Shockley-Read-Hall recombination and surface recombination contributions in complete recombination. The maximum attainable $\eta$ value for these parameters is $29.2 \%$. If the solar cell thickness decreases from 200 to $50 \mu \mathrm{m}$, the limiting $\eta$ value increases to $30.4 \%$.

Next, let us to write theoretical equations for opencircuit voltage and photoconversion efficiency versus base doping level for $25 \%$-effective record silicon solar cell [23], as well as for silicon solar cells with surface recombination, minimized by the $\alpha-\mathrm{Si}: \mathrm{H}-\mathrm{Si}$ heterojunctions [24, 25] using the above formalism. First, the recombination parameters for $V_{O C}$ of $706 \mathrm{mV}$ obtained in [23] are calculated. They are determined from the curve 3 in Fig. 13 intersection with the experimental value $706 \mathrm{mV}$. Next, we substitute the parameters, for which this coincidence was obtained, and the experimental short-circuit current density value equal to $42.7 \mathrm{~mA} / \mathrm{cm}^{2}$, in the expression for $\eta$. As a result, $\eta=25.3 \%$ is achieved. The relevant parameters for the $\alpha-\mathrm{Si}: \mathrm{H}-\mathrm{Si}$ heterojunction solar cells $[24,25]$ were calculated in the similar manner. The final results are presented in Table 3. It can be seen from the table that the calculated open-circuit voltage values coincide with the experimentally obtained in all the cases. The matching error between the calculated and the experimental $\eta$ values is less than $1 \%$. These results demonstrate the adequacy of the theoretical model proposed in this paper relative to the experimental results obtained in [23-25].

In conclusion, we compare the results obtained in this work with the results of the research [17]. In this paper, Fig. 8 shows the results of the open-circuit voltage calculation depending on the silicon solar cell thickness taking into account radiative recombination and interband Auger recombination. These dependences are calculated using expressions (21) and (15). First, let us correct the parameters to those of [17]. Thus, we assume that the intrinsic charge carriers concentration in silicon (for $T=300 \mathrm{~K}$ ) equals to $1.45 \cdot 10^{10} \mathrm{~cm}^{-3}$, and the radiative recombination coefficient equals to $2.5 \cdot 10^{-15} \mathrm{~cm}^{3} / \mathrm{s}$ [17]. Let us also omit the 
$2.5 \cdot 10^{-22} / \Delta p^{0.5}$ term in $C_{n}$ that is absent in [17]. The comparison of the $V_{O C}(d)$ dependences calculated using the formulae (15) and (21) of our work (Fig. 16, curve 1) with those achieved in [17] (Fig. 8) (see Fig. 16 of this paper, curve 2) show a good agreement. This figure also shows the $V_{O C}(d)$ curves constructed using the formulae (15) and (21) of this paper and the parameters of silicon from $[19,20]$ (curve 3 ). There is a good coincidence of the curves (1) and (3), which are practically the same for the actual silicon solar cell thickness (close to $100 \mu \mathrm{m}$ ), although they are different for small and large SC thicknesses. A little higher $V_{O C}(d)$ values at small thickness values are associated with the use of lower hole and electron concentrations in silicon equal to $8.5 \cdot 10^{9} \mathrm{~cm}^{-3}$. Lower $V_{O C}(d)$ values at large thickness values are explained by the greater radiative recombination parameter at room temperature $\left(6 \cdot 10^{-15} \mathrm{~cm}^{3} / \mathrm{s}\right)$ and by taking into account the additional term in the relation for $C_{n}$.

The limiting silicon solar cells photoconversion efficiency in our work correlates with that obtained in [17], and is consistent with the conclusions of [26]. However, the photon recycling for calculating the silicon solar cells parameters (including the maximum possible and record) can be neglected, as shown by the results of our analysis, in contrast to [4, 17]. As a result, the calculation is greatly simplified and can be performed in the traditional approximations.

\section{Conclusions}

An approach to optimize SC based on direct- and indirect-gap semiconductors in order to obtain the maximal photoconversion efficiency has been proposed.

Analysis shows the secondary role of photon recycling even in highly reflective structures for commercial GaAs parameters, particularly typical Shockley-Read-Hall recombination lifetime $\tau_{S R}$ values. The open-circuit voltage $V_{O C}$ enhancement by the doping level optimization has been shown to be much more critical for photoconversion efficiency growth. The optimal doping level of about $10^{17} \mathrm{~cm}^{-3}$ has been determined. The maximal photoconversion efficiency $\eta_{\max }$ for such condition is near $27 \%$.

The doping level and polarity type influence on the GaAs absorption coefficient and photoconversion efficiency has been studied. It has been shown that the conduction band filling with electrons and the respective increase in the donor doping level (Burstein-Moss effect for $n$-type semiconductor) leads to a decrease in the short-circuit current. The fundamental band edge blur increase (Urbach shift) leads to an increase in the shortcircuit current for $p$-type semiconductor.

The influence of the ratio $b$ of the cross-sections for electrons and holes captured by the deep recombination levels on the open-circuit voltage and, consequently, the photoconversion efficiency has been demonstrated.
The developed formalism allows to analyze and to optimize SC parameters for other direct-gap semiconductors, particularly in $\mathrm{A}^{3} \mathrm{~B}^{5}$ semiconductors.

The open-circuit voltage and photoconversion efficiency generation features for monocrystalline silicon solar cells with more than 1 ms Shockley-ReadHall lifetime have been considered.

A formalism describing quantitatively the experimental results for high-efficiency silicon solar cells using various surface recombination velocity minimization techniques has been also proposed.

The approach of this research allows to predict the expected solar cell (for both direct- and indirect-gap semiconductors) characteristics, if material parameters are known.

\section{References}

1. O.D. Miller, E. Yablonovitch and S.R. Kurtz, Strong internal and external luminescence as solar cells approach the Shockley-Queisser limit // IEEE J. Photovoltaics, 2(3), p. 303-311 (2012).

2. O. Miller Photonic Design: From Fundamental Solar Cell Physics to Computational Inverse Design, Doctoral dissertation. University of California, Berkeley, USA, 2012, 137 p.

3. I. Schnitzer, E. Yablonovitch, C. Caneau and T.J. Gmitter, Ultrahigh spontaneous emission quantum efficiency, 99.7\% internally and $72 \%$ externally, from AIGaAs/GaAs/AIGaAs double heterostructures // Appl. Phys. Lett. 62(2), p. 131133 (1993).

4. D. Ding, S.R. Johnson, S.-Q. Yu, S.-N. Wu and Y.-H. Zhang, A semi-analytical model for semiconductor solar cells // J. Appl. Phys. 110, 123104 (2011).

5. H.C. Casey, D.D. Sell, and K.W. Wecht, Concentration dependence of the absorption coefficient for n- and p-type GaAs between 1.3 and $1.6 \mathrm{eV} / /$ J. Appl. Phys. 46(1), p. 250-257 (1975).

6. W. Van Roosbroeck, W. Shokley, Photon-radiative recombination of electrons and holes in germanium // Phys. Rev. 94(6), p. 1558-1560 (1954).

7. G.B. Lush, H.F. MacMillan, B.M. Keyes, D.H. Levi, R.K. Ahrenkiel, M.R. Melloch and M.S. Lundstrom, A study of minority carrier lifetime versus doping concentration in n-type GaAs grown by metalorganic chemical vapor deposition // J. Appl. Phys. 72(4), p. 1436-1442 (1992).

8. Hiroshi Ito, Tomofumi Furuta and Tadao Ishibashi, Minority electron lifetimes in heavily doped p-type GaAs grown by molecular beam epitaxy // Appl. Phys. Lett. 58(25), p. 2936-2938 (1991).

9. A.L. Fahrenbruch and R.H. Bube. Fundamentals of Solar Cells. Photovoltaic Solar Energy Conversion. New York, 1983.

10. H. Nie, B.M. Kayes and I. Kizilyalli, Optoelectronic devices including heterojunction. EP Patent 2,450,956. 
11. A.P. Gorban, A.V. Sachenko, V.P. Kostylyov and N.A. Prima, Effect of excitons on photoconversion efficiency in the $\mathrm{p}^{+}-\mathrm{n}-\mathrm{n}^{+}-$and $\mathrm{n}^{+}-\mathrm{p}-\mathrm{p}^{+}$-structures based on single-crystalline silicon // Semiconductor Physics, Quantum Electronics and Optoelectronics, 3(3), p. 322-329 (2000).

12. S.M. Sze and Kwok K. Ng, Physics of Semiconductor Devices, $3^{\text {rd }}$ Ed. John Wiley and Sons, 2007.

13. A.P. Gorban, V.P. Kostylyov, A.V. Sachenko, O.A. Serba, I.O. Sokolovskyi, V.V. Chernenko, Effect of floating $p$ - $n$ junctions on the efficiency of silicon back side contact solar cells // Ukr. J. Phys. 55(7), p. 783-790 (2010).

14. B.M. Kayes, H. Nie, R. Twist, S.G. Spruytte, F. Reinhardt, I.C. Kizilyalli, G.S. Higashi, 27.6\% conversion efficiency, a new record for singlejunction solar cells under 1 sun illumination // Photovoltaic Specialists Conference (PVSC), 2011 37th IEEE, p. 000004-000008, June 19-24, 2011.

15. P.A. Folkes, B. Connelly and F. Towner, Minority carrier lifetime and interfacial recombination velocity in GaAs/AlGaAs double heterostructures // ARL-TR-6186 Report.

16. R.I. Jioev, K.V. Kavokin, Optical measurement of diffusion length and electron surface recombination velocity in the $p$-GaAs layers // Fizika i tekhnika poluprovodnikov, 33(10), p. 2928-2931 (1991), in Russian.

17. T. Tiedje, E. Yablonovitch, G.D. Cody and B.J. Brooks, Limiting efficiency of silicon solar cells // IEEE Trans. on Electron Devices, 31(5), p. 711-716 (1984).

18. T. Trupke, J. Zhao, A. Wang, R. Corkish, M.A. Green, Very efficient light emission from bulk crystalline silicon // Appl. Phys. Lett. 82, p. 2996-2998 (2003).
19. A.V. Sachenko, A.P. Gorban, V.P. Kostylev, I.O. Sokolovskii, Quadratic recombination in silicon and its influence on the bulk lifetime // Semiconductors, 41(3), p. 281-284 (2007).

20. A.V. Sachenko, A.P. Gorban, V.P. Kostylyov, I.O. Sokolovsky, The radiative recombination coefficient and the internal quantum yield of electroluminescence in silicon // Semiconductors, 40(8), p. 884-889 (2006).

21. E. Yablonovitch and T. Gmitter, Auger recombination in silicon at low carrier densities // Appl. Phys. Lett. 49(10), p. 587-589 (1986).

22. E. Yablonovitch, D.L. Allara, C.C. Chang, T. Gmitter and T.B. Bright, Unusually low surfacerecombination velocity on silicon and germanium surfaces // Phys. Rev. Lett. 57(2), p. 249-252 (1986).

23. M.A. Green, The path to $25 \%$ silicon solar cell efficiency: History of silicon cell evolution // Progr. Photovolt.: Res. Appl. 17(3), p. 183-189 (2009).

24. T. Sawada, N. Terada, Sadaji Tsuge, Baba Toshiaki, T. Takahama, Kenichiro Wakisaka, Shinya Tsuda, Shoichi Nakano, High-efficiency a$\mathrm{Si}$ /c-Si heterojunction solar cell // 1994 IEEE First World Conference on Photovoltaic Energy Conversion, Conference Record of the Twenty Fourth IEEE Photovoltaic Specialists Conference, p.1219-1226 (1994).

25. A. Yano, S. Tohoda, K. Matsuyama, Y. Nakamura, T. Nishiwaki, K. Fujita, M. Taguchi, E. Maruyama, $24.7 \%$ record efficiency HIT® solar cell on thin silicon wafer // 28th European Photovoltaic Solar Energy Conference and Exhibition, September 30 October 4, 2013, Paris, France, p. 748-751.

26. R.M. Swanson, Approaching the $29 \%$ limit efficiency of silicon solar cells // 20 European Photovoltaic Conference, June 6-10, 2005, Barcelona, Spain, p. 584-589. 